\title{
Melatonin suppresses neuronal regeneration following neuronal degeneration in the hippocampal dentate gyrus
}

\author{
Masanori Yoneyama*, Shigeru Hasebe, Taro Yamaguchi, Yusuke Onaka and Kiyokazu Ogita \\ Laboratory of Pharmacology, Setsunan University Faculty of Pharmaceutical Sciences, Japan
}

\begin{abstract}
Here, we evaluated the effect of in vivo treatment with melatonin on neuronal regeneration after neuronal loss in the dentate gyrus of TMT-treated mouse, which is an in vivo model for neuronal repair following neuronal loss in the hippocampal dentate gyrus.Fourteen-days treatment with melatonin ( $50 \mathrm{mg} / \mathrm{kg}$, i.p.) dramatically suppressed the increase in the number of 5-bromo-2'-deoxyuridine (BrdU)-incorporating cells and NeuN-positive BrdU-incorporated cells generated after neuronal loss in the dentate gyrus of TMT treated animals on day 30 post-TMT treatment. Melatonin was effective in decreasing the level of ionized calcium-binding adapter molecule 1 expressed in the dentate gyrus of TMT-treated animals.Our data suggest that that melatonin suppresses neuronal repair following neuronal loss in the dentate gyrus.
\end{abstract}

\section{Introduction}

Adult neurogenesis occurs in 2 regions in the brain, i.e., the hippocampal dentate gyrus and olfactory bulb [1,2]. In adult neurogenesis, proliferation and differentiation of neural stem/ progenitor cells are enhanced for replacing the cells damaged/lost following neural injury with new neuronal and glial cells. For instance, brain ischemia enhances neurogenesis in the sub granular zone (SGZ) of the dentate gyrus and the subventricular zone of lateral ventricle [3]. Ischemia-induced neurogenesis is considered as being a compensatory mechanism in response to neuronal damage in the brain. Therefore, treatment that enhances the neurogenesis process has been thought to be a beneficial therapy for neuronal injury or neurodegenerative disorders.

Our previous studies demonstrated that a single systemic treatment of mice with trimethyltin chloride (TMT) causes neuronal loss in restricted brain regions, including the dentate gyrus, olfactory bulb, anterior olfactory nucleus, and frontal cerebral cortex [4,5]. Following TMT-induced neuronal loss in the dentate gyrus and olfactory bulb, neurogenesis was enhanced through proliferation of the neural stem/ progenitor cells and neuronal precursor cells in each of these brain regions $[6,7]$. These findings indicate that the TMT-treated mouse is a very attractive model for studies of neuronal self-repair (regeneration) following neuronal loss in the dentate gyrus.

Melatonin mediates its effects through 2 subtypes of receptor including MT1 and MT2 [8], and regulates antioxidant enzymes [9] by switching on/off intracellular signaling cascades [10], as well as by scavenging oxygen free radical [11]. In addition, melatonin promotes neurogenesis under diverse conditions, such as ovariectomy, pinealectomy, aging or circadian disruption [12]. However, it has been not evaluated if melatonin affects neurogenesis following crucial neuronal loss in the hippocampal dentate gyrus. Therefore, the present study was designed to elucidate the effect of melatonin on neuronal regeneration following neuronal loss in the dentate gyrus in the TMTtreated mouse.

\section{Materials and methods}

The protocol used here met the guidelines of The Japanese Society for Pharmacology and was approved by the Committee for Ethical Use of Experimental Animals at Setsunan University. Adult male Std-ddY mice at 5-6 weeks of age were intraperitoneally injected with TMT (2.9 $\mathrm{mg} / \mathrm{kg}$ ) dissolved in phosphate-buffered saline (PBS) for preparing the mouse model of loss/self-repair in the hippocampal dentate gyrus. Melatonin $(50 \mathrm{mg} / \mathrm{kg}$ ) was dissolved in PBS and intraperitoneally injected into the mice once a day for the desired number of days, starting on day 2 post-TMT treatment. To label mitotic cells, mice were administrated a single series of two consecutive injections of BrdU (50 $\mathrm{mg} / \mathrm{kg}$, i.p., dissolved in PBS) at a $12-\mathrm{hr}$ interval on day 2 post-TMT treatment. These mice were then returned to their home cages until the time of decapitation.

We divided the animals into 4 different groups for the experiments, PBS and vehicle for melatonin-treated group (naïve/vehicle), PBS and melatonin-treated group (naïve/melatonin), TMT and vehicle for melatonin-treated group (TMT/vehicle), TMT and melatonin-treated group (TMT/melatonin). To examine the effect of chronic treatment with melatonin on the proliferation/survival and differentiation of neural progenitor cells generated following TMT-induced neuronal loss in the dentate gyrus, we carried out animals were administrated either melatonin or vehicle daily on days 2-15 post-treatment with PBS or TMT and then decapitated on day 30 post-treatment with PBS or TMT (Figure 1).

Histological determination of 5-bromo-2'-deoxyuridine (BrdU)-

Correspondence to: Masanori Yoneyama, Laboratory of Pharmacology, Setsunan University Faculty of Pharmaceutical Sciences, 45-1 Nagaotoge-cho, Hirakata, Osaka 573-0101, Japan, Tel: 81-(0)72-866-3111; Fax: 81-(0)72-866-3111; E-mail: m-yone@pharm.setsunan.ac.jp

Key words: melatonin, hippocampal dentate gyrus, neurogenesis

Received: March 10, 2017; Accepted: April 26, 2017; Published: April 30, 2017 
incorporated cells and NeuN-positive cells, immunostaining was performed using each antibody against BrdU (Abcam, Cambridge, MA, USA) and NeuN (Chemicon, Temecula, CA, USA) in sagittal sections of paraffin- embedded brain as described previously [13]. For immunoblot analysis, protein samples were prepared from the hippocampal dentate gyrus of each animals according to the method described in the previous report [14]. Immunoblot assays were carried out by using primary antibodies against ionized calcium-binding adapter molecule 1 (Iba1, Wako Pure Chemical Industries Ldt., Osaka, Japan) and glyceraldehyde-3-phosphate dehydrogenase (GAPDH, Santa Cruz Biotechnology Inc., Santa Cruz., CA, USA) as described previously [14]. The statistical significance was determined by use of the two-tailed Student t-test, one-way ANOVA with Bonferroni/ Dunnett post hoc test, or two-way repeated measure ANOVA.

\section{Results}

\section{Effect of melatonin on proliferation/survival of cells generated following neuronal loss in the dentate gyrus}

To evaluate the effect of melatonin on proliferation and survival of newly-generated cells in the dentate gyrus, we determined the number of BrdU-incorporated cells in the dentate granule cell layer and subgranular zone of naïve or TMT-treated animals treated with melatonin for 14 days (Figure 2). In animals treated with vehicle alone, the number of BrdU-incorporated cells in these regions were dramatically increased in TMT-treated animals compared with naïve animals. Melatonin completely abolished to increase the number of

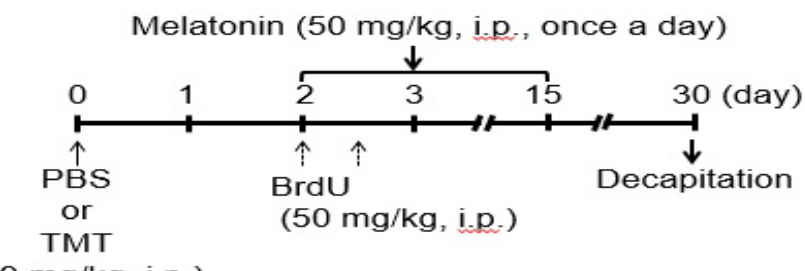

$(2.9 \mathrm{mg} / \mathrm{kg}$, i.p. $)$

Figure 1. Experimental schedule.

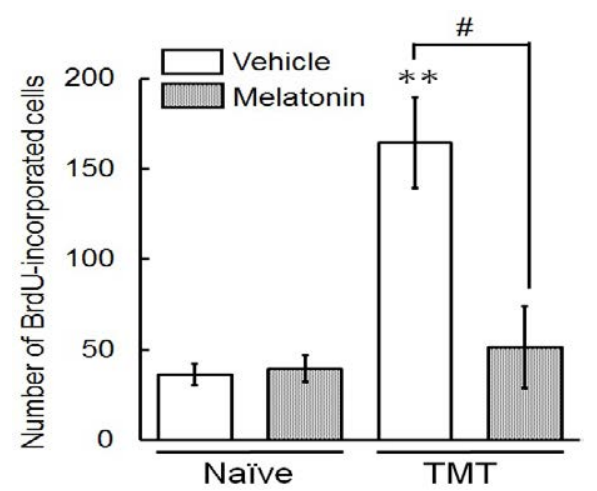

Animals were given either PBS ("Naïve") or TMT ( $2.9 \mathrm{mg} / \mathrm{kg}$, i. p., "TMT") and then were given $\mathrm{BrdU}(50 \mathrm{mg} / \mathrm{kg}$, i. p.) on day 2 post-TMT treatment and then decapitated on day 30 post-TMT treatment for preparation of sagittal hippocampal sections, which were then stained with antibodies BrdU. The graph shows the number of BrdU-incorporated cells in the granule cell layer and subgranular zone of 4 animal groups (naïve/vehicle, naïve/ melatonin, TMT/vehicle, TMT/melatonin). Values are expressed as the mean \pm S.E.M. calculated from 4 separate experiments. ${ }^{* *} P<0.01$, significantly different from each value obtained for the naïve animals. ${ }^{~} P<0.05$, significant difference the values obtained between animals treated with vehicle and melatonin.

Figure 2. Effect of melatonin on neuronal proliferation/survival of cells generated followingneuronal loss in the dentate gyrus.
BrdU-incorporated cells in there of TMT-treated animals. In naïve animals, however, melatonin was ineffective in affecting the number of BrdU-incorporated cells in these regions.

Effect of melatonin on differentiation of $\mathrm{BrdU}(+)$ cells generated following neuronal loss in the dentate gyrus

To assess the fate of newly-generated cells in the dentate gyrus following neuronal loss, we carried out double-labeling of BrdU and NeuN on day 30 post-treatment with PBS (naïve) or TMT (Figure 3). Comparing cells positive for both $\mathrm{NeuN}$ and BrdU between the naïve and TMT-treated animals, no significant change in the numbers of those cells was observed in the granule cell layer and SGZ of animals treated with vehicle alone. Expectedly, treatment with melatonin markedly decreased the number of NeuN-positive BrdU-incorporated cells (newly-generated neurons) in these resions of TMT-treated animals. However, melatonin had no effect on the number of NeuNpositive BrdU-incorporated cells in there of the naïve animals.

\section{Effect of melatonin on the expression of Ibal there following neuronal loss in the dentate gyrus.}

Adult neurogenesis is regulated by numerous endogenous factors produced during neurodegeneration [15]. Neurogenesis following neurodegeneration are known to be enhanced by activated microglia through regulation of proliferation and survival of neural stem/ progenitor cells by releasing various soluble factors [16,17]. Here, we examined the effect of melatonin on expression of Ibal in the dentate gyrus on days 3 and 5 post-TMT treatment (Figure 4). Melatonin had the ability to significantly decrease the expression level of Ibal in the dentate gyrus on day 5 post-TMT treatment.

\section{Discussion}

The main finding of this study was that chronic melatonin treatment dramatically suppressed neuronal differentiation following neuronal loss probably through abolished activation of microglia in the dentate gyrus. The process of adult neurogenesis in the hippocampus is accomplished in at least 3 steps including the proliferation, migration, and survival/differentiation of neural stem/progenitor celss. To address (a)

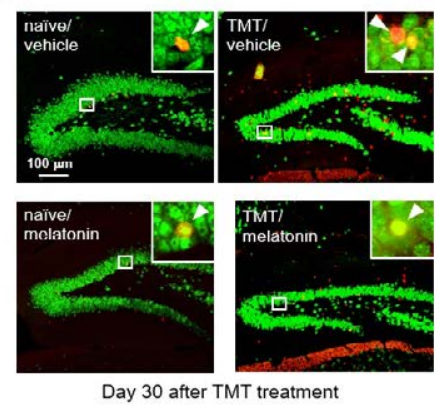

(b)

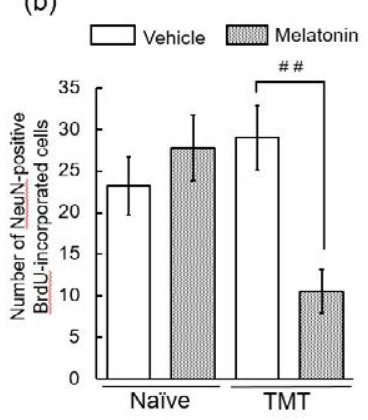

Animals were given either PBS ("Naïve") or TMT ( $2.9 \mathrm{mg} / \mathrm{kg}$, i. p., "TMT") and then were given BrdU $(50 \mathrm{mg} / \mathrm{kg}$, i. p.) on day 2 post-TMT treatment and then decapitated on day 30 post-TMT treatment for preparation of sagittal hippocampal sections, which were stained with antibodies NeuN and BrdU. (a) Typical micrographs show NeuN-positive cells (green) and BrdU-incorporated cells (red) in the dentate gyrus of the 4 animal groups. NeuN-positive BrdU-incorporated cells are denoted as yellow color. (b) The graph shows the number of NeuN-positive BrdU-incorporated cells in the GCL and SGZ of the 4 animal groups. Values are expressed as the mean \pm S.E.M. calculated from 4 separate experiments. ${ }^{\#} P<0.01$, significant difference the values obtained between animals treated with vehicle and melatonin.

Figure 3. Effect of melatonin on neuronal differentiation of cells generated following neuronal loss in the dentate gyrus. 


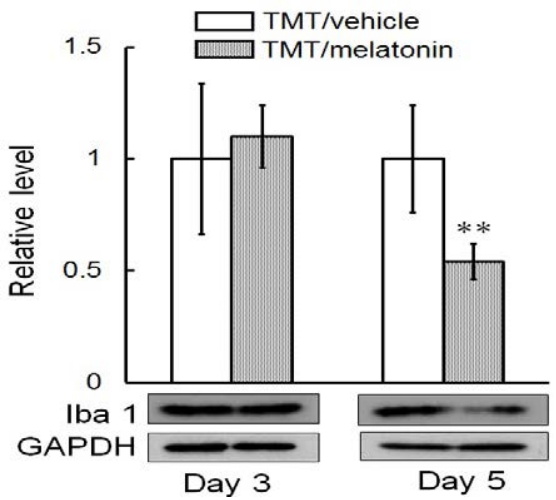

Animals were administrated either melatonin or vehicle on day 2 post-treatment with TMT and then decapitated on days 3 and 5 for preparation of the tissue lysates, which were subjected to immunoblot analysis. Values are expressed as the mean \pm S.E.M. calculated from 4 separate experiments. ${ }^{* *} P<0.01$, significantly different from the control value obtained in the vehicle group of the impaired animals.

Figure 4. Effect of melatonin on Ibal level expressed in the dentate gyrus following neuronal loss.

the fate of newly generated cells on day 30 following neuronal loss in the dentate gyrus, we evaluated the effect of the chronic treatment with melatonin on the BrdU-incorporating cells positive for NeuN. The current data show that the chronic treatment with melatonin suppressed neuronal differentiation of the newly-generated cells in the dentate gyrus in TMT-produced the dentate neuronal loss/repair model. These newly-generated neuronal cells were located predominantly in the granule cell layer, but not in the molecular layer and hilus of the dentate gyrus. These data are possible that melatonin suppressed these newlygenerated cells to be survival and/or to differentiate into neuronal cells. The finding that melatonin had no significant effect on the dentate newly-generated neuronal cells in naive animals leads to propose that the melatonin-induced suppression of hippocampal neurogenesis is selective in affecting only the impaired dentate gyrus.

How does melatonin suppressed neurogenesis in the injured dentate gyrus? One possibility is that melatonin suppresses activation of microglia in the injured dentate gyrus. Indeed, the present study showed that melatonin reduced the level of Ibal, which is a marker of microglia, in the dentate gyrus following dentate neuronal loss in TMT-treated animals. Although the mechanisms underlying suppression by melatonin of microglia activation are not fully established, a previous study demonstrated that melatonin modulates some transcription factors in microglia and then decreases the release of pro-inflammatory cytokines by these cells [18]. Further evidence for involvement of microglia in neurogenesis comes from a previous report that suppression of microglia activation with minocycline reduces neurogenesis after middle cerebral artery occlusion [19]. These previous findings support the proposition that melatonin suppresses survival and/or differentiation of newly-generated cells into neuronal cells probably through inactivation of microglia in the hippocampal dentate gyrus.

\section{Conclusion}

We provided evidence for the ability of melatonin to prevent proliferation, survival and/or neuronal differentiation of neural stem/ progenitor cells newly-generated in the dentate gyrus following dentate neuronal loss caused by treatment with TMT. The melatonin-induced events are probably derived from abolishment of microglia activation following neuronal injury. Hence, it is possible that the suppression by melatonin receptor antagonists or inhibitors for melatonin synthesis of melatonin signals is capable of facilitating neurogenesis after neuronal damage in the dentate gyrus.

\section{Acknowledgments}

The authors have no conflicts of interest. This work was supported in part by grants-in-aid for scientific research to M.Y. from the Ministry of Education, Culture, Sports, Science and Technology, Japan.

\section{Conflict of interest}

The authors have conflicts of interest.

\section{References}

1. Kuhn HG, Dickinson-Anson H, Gage FH (1996) Neurogenesis in the dentate gyrus of the adult rat: age-related decrease of neuronal progenitor proliferation. J Neurosci Res 16: 2027-2033. [Crossref]

2. Taupin P1, Gage FH (2002) Adult neurogenesis and neural stem cells of the central nervous system in mammals. J Neurosci Res 69: 745-749. [Crossref]

3. Wójcik-Stanaszek L, Sypecka J, Szymczak P, Ziemka-Nalecz M, Khrestchatisky M, et al. (2011) The potential role of metalloproteinases in neurogenesis in the gerbil hippocampus following global forebrain ischemia. PLoS One 6: e22465. [Crossref]

4. Ogita K, Nitta Y, Watanabe M, Nakatani Y, Nishiyama N, et al. (2004) In vivo activation of c-Jun N-terminal kinase signaling cascade prior to granule cell death induced by trimethyltin in the dentate gyrus of mice. Neuropharmacology 47:619-630. [Crossref]

5. Kawada K, Yoneyama M, Nagashima R, Ogita K (2008) In vivo acute treatment with trimethyltin chloride causes neuronal degeneration in the murine olfactory bulb and anterior olfactory nucleus by different cascades in each region. $J$ Neurosci Res $86: 1635-1646$. [Crossref]

6. Ogita K, Nishiyama N, Sugiyama C, Higuchi K, Yoneyama M, et al. (2005) Regeneration of granule neurons after lesioning of hippocampal dentate gyrus: evaluation using adult mice treated with trimethyltin chloride as a model. $J$ Neurosci Res 82:609-621. [Crossref]

7. Yoneyama M, Kawada K, Ogita K (2010) Enhanced neurogenesis in the olfactory bulb in adult mice after injury induced by acute treatment with trimethyltin. $J$ Neurosci Res 88: 1242-1251. [Crossref]

8. Dubocovich ML (1988) Luzindole (N-0774): a novel melatonin receptor antagonist. $J$ Pharmacol Exp Ther 246: 902-910. [Crossref]

9. Tomás-Zapico C, Coto-Montes A (2005) A proposed mechanism to explain the stimulatory effect of melatonin on antioxidative enzymes. J Pineal Res 39: 99-104. [Crossref]

10. Radogna F, Diederich M, Ghibelli L (2010) Melatonin: a pleiotropic molecule regulating inflammation. Biochem Pharmacol 80: 1844-1852. [Crossref]

11. Manchester MLC, Coto-Montes A, Boga JA, Andersen LP, Zhou Z, et al. (2015) Melatonin: an ancient molecule that makes oxygen metabolically tolerable. $J$ Pineal Res 59:403-419.

12. Mueller AD, Mear RJ, Mistlberger RE (2011) Inhibition of hippocampal neurogenesis by sleep deprivation is independent of circadian disruption and melatonin suppression. Neuroscience 193:170-181. [Crossref]

13. Yoneyama M, Shiba T, Haebe S, Umeda K, Yamaguchi T, et al. (2014) Lithium promotes neuronal repair and ameliorates depression-like behavior following trimethyltininduced neuronal loss in the dentate gyrus. PLOS One 4: e87953. [Crossref]

14. Yoneyama M, Haebe S, Shiba T, Yamaguchi T, Ogita K (2015) Beneficial effect of cilostazol-mediated neuronal repair following trimethyltin-induced neuronal loss in the dentate gyrus. J Neurosci Res 93:55-66. [Crossref]

15. Yoneyama M, Shiba T, Hasebe S, Ogita K (2011) Adult neurogenesis is regulated by endogenous factors produced during neurodegeneration. J Pharmacol Sci 115: 425432. [Crossref]

16. Walton NM, Sutter BM, Laywell ED, Levkoff LH, Kearns SM, et al. (2006) Microglia instruct subventricular zone neurogenesis. Glia 54: 815-825. [Crossref]

17. Battista D, Ferrari CC, Gage FH, Pitossi FJ (2006) Neurogenic niche modulation by activated microglia: transforming growth factor beta increases neurogenesis in the adult dentate gyrus. Eur J Neurosci 23: 83-93. [Crossref] 
18. Jiang C, Ting AT, Seed B (1998) PPAR-gamma agonists inhibit production of monocyte inflammatory cytokines. Nature 391: 82-86. [Crossref]
19. Kim BJ, Kim MJ, Park JM, Lee SH, Kim YJ, Ryu S, Kim YH, Yoon BW (2009) Reduced neurogenesis after suppressed inflammation by minocycline in transient cerebral ischemia in rat. J Neurol Sci 279: 70-75. [Crossref]

Copyright: $(02017$ Yoneyama M. This is an open-access article distributed under the terms of the Creative Commons Attribution License, which permits unrestricted use, distribution, and reproduction in any medium, provided the original author and source are credited. 